\title{
A case against the specificity of "cardiac" troponin-T
}

\author{
M Mahalingam, M E Ottlinger
}

\begin{abstract}
A case of a spurious rise in cardiac troponin-T in an 85 year old Caucasian man with myelodysplastic syndrome and multiple malignancies but with intact cardiac and renal function is reported. The patient presented to the accident and emergency department with fever and chest pain. Inconsistent laboratory findings in biochemical markers diagnostic of myocardial infarction were observed. Discrepant findings included a rise in the concentration of the cardiac specific marker troponin- $T$ in the absence of an increase in creatine kinase (CK) isoenzyme MB activity. Somewhat surprisingly, there was a significant and consistent increase in $\mathrm{CK}$ isoenzyme $\mathrm{BB}$ activity. Awareness of the increase in troponin-T concentrations in patients with multiple clinical non-cardiac problems may prevent an erroneous diagnosis of myocardial infarction and avert institution of unduly aggressive treatment.

$(\mathcal{F}$ Clin Pathol 1996;49:766-767)
\end{abstract}

Keywords: troponin-T, CK-MB, CK-BB, intact renal function.

\section{Assay details}

CARDIAC TROPONIN-T ASSAY

All measurements of cardiac troponin- $T$ were made with a commercially available enzyme immunoassay kit (Boehringer Mannheim, Indianapolis, Indiana, USA). Briefly, the assay is based on a single step sandwich principle with streptavidin coated tubes as the solid phase and two monoclonal anti-human cardiac troponin- $T$ antibodies. The intra-assay coefficient of variation was $<2 \%$.

\section{CK-MB MASS ASSAY}

Creatine kinase $(\mathrm{CK})$ isoenzyme $\mathrm{MB}$ mass was measured using a microparticle enzyme immunoassay (Abbott Systems, Chicago, Illinois, USA). The assay is based on a two site immunometric sandwich method with two monoclonal antibodies - a capture antibody specific for CK-MB and an enzyme labelled (alkaline phosphatase) antibody. The intra-assay coefficient of variation was $<5 \%$.

\section{CK-MB ELECTROPHORESIS ASSAY}

The percentage of CK-MB in a given sample was measured by electrophoresis (Helena Laboratories, Beaumont, Texas, USA). Briefly, the isoforms of CK are first separated according to their electrophoretic mobility. The sepa- rated isoenzymes are then incubated with specific reagents to visualise the separated isoforms. The intra-assay coefficient of variation was $<5 \%$.

\section{Case report}

An 85 year old Caucasian man, with a history of bone marrow biopsy confirmed myelodysplastic syndrome (MDS) and prostate and colon carcinoma, was brought to the accident and emergency department after his family found him "looking unwell". On admission, he complained of left sided chest pain. Physical examination revealed that he was febrile (temperature $40.6^{\circ} \mathrm{C}$ ). The patient's pulse rate was 120 /minute, respiratory rate was 28 /minute and his baseline blood pressure was $120 / 90$. He seemed to be in moderate respiratory distress. Auscultation revealed that the patient had pulmonary oedema. The following were the findings from cardiac examination: apical impulse in the sixth anterior axillary line, loud first and second heart sound, regular heart rate and rhythm, and absence of a murmur. A chest radiograph showed a normal sized heart, bilateral pleural effusion with a questionable lingular infiltrate which was obscured by the effusion. A 12-lead electrocardiogram (ECG) showed evidence of left ventricular hypertrophy, atrial fibrillation and right bundle branch block. The tracing was unchanged from that taken a month earlier.

Table 1 summarises the laboratory findings. $\mathrm{LDH}$ fractionation (\%) indicated the following: on admission LD1 13.2, LD2 30.9, LD3 20.7, LD4 11.2, LD5 24.1; and 12 hours later LD1 11.7, LD2 32.3, LD3 22.2, LD4 11.6, LD5 23.2. CK fractionation (\%) indicated the N following (both by the mass assay and by electrophoresis): on admission CK-MM 92.5, CK-MB 0, CK-BB 7.5; and 12 hours later CK-MM 92, CK-MB 0, CK-BB 8.

\section{Discussion}

Troponin- $\mathrm{T}$, the tropomyosin binding, cardiac specific subunit of the troponins, is one of the best known examples of a non-cytoplasmic marker of myocardial infarction. ${ }^{1}$ However, its specificity has not been fully delineated in noncardiac diseases. There are published reports to indicate that elevations in troponin- $T$ may be seen in a patients with polymyositis/ dermatomyositis and also in patients with acute muscle disease, trauma patients and patients with rhabdomyolysis. ${ }^{2}$ Reports, including a recent one, indicate that spurious rises in troponin- $T$ may be seen in end-stage 
Table 1 Summary of laboratory findings

\begin{tabular}{llll}
\hline Test & $\begin{array}{l}\text { Values on } \\
\text { admission }\end{array}$ & $\begin{array}{l}\text { Values 12 hours } \\
\text { after admission }\end{array}$ & $\begin{array}{l}\text { Reference range } \\
\text { (SI units) }\end{array}$ \\
\hline WBC & 18.9 & 29.0 & $4.8-10.8 \times 10^{9} / 1$ \\
Haemoglobin & 10.1 & 9.5 & $14-17 \mathrm{~g} / \mathrm{dl}$ \\
Platelets & 238 & 175 & $150-450 \times 10^{9} / 1$ \\
Sodium & 133 & 135 & $134-144 \mathrm{mmol} / 1$ \\
AST & 101 & 342 & $8-37 \mathrm{U} / 1$ \\
ALT & 75 & 248 & $0-39 \mathrm{U} / 1$ \\
Potassium & 4.6 & 4.7 & $3.7-4.9 \mathrm{mmol} / 1$ \\
Creatinine & 105 & 106 & $53-106 \mu \mathrm{mol} / 1$ \\
Blood urea nitrogen & 8.6 & 8.9 & $2.9-8.2 \mathrm{mmol} / 1$ \\
Total bilirubin & 46.2 & 42.8 & $2-21 \mu \mathrm{mol} / 1$ \\
LDH & 1118 & 1215 & $102-228 \mathrm{U} / 1$ \\
CK & 1121 & 660 & $0-183 \mathrm{U} / 1$ \\
Troponin-T & 5.5 & 5.4 & $0-0.1 \mathrm{ng} / 1$ \\
\hline
\end{tabular}

WBC = white blood cells; AST = aspartate aminotransferase; ALT = alanine aminotransferase; $\mathrm{LDH}=$ lactate dehydrogenase.

renal disease. ${ }^{34}$ Ours is the first report to indicate that such spurious rises may also be seen in the presence of intact renal function.

As there were no clinical electrocardiograph tracings in our patient suggestive of myocardial infarction, we believe that the increased concentrations of troponin- $T$ were an erroneous indicator of myocardial infarction. There are two possible reasons for the rise in troponin- $T$ concentrations: firstly, as a result of the existence of minor myocardial injuries undetectable in the ECG. ${ }^{1}$ Lack of an increase in the CK-MB fraction could be explained by the fact that cardiac troponin- $T$ is known to have a higher sensitivity and therefore a lower diagnostic efficiency than $\mathrm{CK}-\mathrm{MB}$ in the diagnosis of myocardial infarction. ${ }^{1}$ A second, alternative explanation, and one that we favour, is that the raised cardiac troponin- $T$ concentration is a consequence of the multiple disorders, septicaemia and widespread malignancy present in our patient. There is unpublished information from retrospective reviews of medical records to indicate that patients positive for troponin- $T$ and negative for
CK-MB have multiple illnesses. As the presence of multiple illnesses is often a complication of and not an uncommon clinical phenomenon in widespread malignant disease, we believe that this anecdotal information may be of use. It is possible that follow up values after the acute period may have provided an additional insight into the cause of the increases observed in the present patient. However, this information was not available as the patient died two days after admission to hospital. Permission to perform a necropsy was not granted and the cause of death was presumed to be overwhelming sepsis.

The CK-BB isoenzyme has been proposed as a tumour marker as it indicates the presence of solid organ malignancies. ${ }^{5}$ There is also an isolated case report of a patient with MDS with an elevated CK-BB fraction. ${ }^{6}$ Thus, the increased CK-BB fraction observed in our patient may be attributable to either or all of the three malignancies.

Increases in cardiac troponin- $T$ concentrations in the absence of diagnostic ECG changes and an increase in the CK-MB fraction support the exclusion of myocardial infarction as a clinical possibility and re-affirm the use of CK-MB as a diagnostic tool in this condition.

1 Bhayana V, Henderson AR. Biochemical markers of myocardial damage. Clin Biochem 1995;28:1-29.

2 Kobayashi S, Tanaka M, Tamura N, Hashimoto H, Hirose S. Serum cardiac troponin $\mathrm{T}$ in polymyositis/dermatomyositis. Lancet 1992;340:726.

3 Wu AH, Valdes R Jr, Apple FS, Gornet T, Stone MA Mayfield-Stokes S, et al. Cardiac troponin-T immunoassay for diagnosis of acute myocardial infarction. Clin Chem 1994;40:900-7.

4 Croitoru M, Taegtmeyer $H$. Spurious rises in troponin- $T$ in end-stage renal disease. Lancet 1995;346:974.

5 Abbott B, Lott JA. Reactivation of serum creatine kinase isoenzyme $\mathrm{BB}$ in patients with malignancies. Clin Chem 1984;30: $1861-3$

6 Crook M, Williams A, Sankarlingam A, Tutt P. Raised concentration of plasma creatine kinase $B B$ isoenzyme in myelodysplasia. f Clin Pathol 1994;47:552-3.

\section{High temperature antigen retrieval and loss of nuclear morphology: a comparison of microwave and autoclave techniques}

Immunocytochemistry and Molecular

Pathology Unit, Department of Pathology, University Hospital of Wales, Heath Park, Cardiff CF4 4XN

Correspondence to: Dr NCA Hunt, Clinical Lecturer Nuffield Department of Pathology and Microbiology, John Radcliffe Hospital, Headington,

Oxford OX3 9DU.

Accepted for publication 4 June 1996

\section{N C A Hunt, R Attanoos, B Jasani}

\begin{abstract}
The use of high temperature antigen retrieval methods has been of major importance in increasing the diagnostic utility of immunocytochemistry. However, these techniques are not without their problems and in this report attention is drawn to a loss of nuclear morphological detail, including mitotic figures, following microwave antigen retrieval. This
\end{abstract}

was not seen with an equivalent autoclave technique. This phenomenon was quantified using image analysis in a group of $B$ cell lymphomas stained with the antibody L26. Loss of nuclear morphological detail may lead to difficulty in identifying cells accurately, which is important in the diagnostic setting-for example, when trying to distinguish a malignant lymphoid infiltrate within a mixed cell popu- 